\title{
CRITICAL SYSTEMS FOR HIGH PEAK POWER STORAGE RING FEL
}

\author{
I.V. Pinayev, G. Detweiler, M. Emamian, N. Hower, M. Johnson, V.N. Litvinenko, O. Oakley, \\ S.H. Park, J. Patterson, G. Swift, Y. Wu, ELL, Durham, NC
}

\section{Abstract}

The Duke storage ring is a facility dedicated for the deep UV free electron laser (FEL) development [1]. It is especially designed for the high peak power applications, having a long optical cavity and large energy acceptance. In this paper we describe FEL gain modulator and optical cavity control system, which are essential for obtaining maximal peak power.

\section{INTRODUCTION}

Performance of the Duke storage ring and OK-4/Duke FEL [2] is shown in Table 1. The reflectivity of mirrors is very essential to the storage ring FELs, but even perfect mirrors do not ensure lasing if the entire optical system is poorly aligned or mechanically unstable.

Table 1: Duke Storage Ring and OK-4/Duke FEL Parameters

Operational Energy [GeV]

$0.25-1.1$

Circumference $[\mathrm{m}]$

107.46

Impedance of ring, $\mathrm{Z} / \mathrm{n},[\Omega]$

2.75

Stored current $[\mathrm{mA}]$ multibunch single bunch

Bunchlength [ps] natural

with $5 \mathrm{~mA}$ in single bunch

Relative Energy spread, $\delta \mathrm{E} / \mathrm{E}$ natural $0.9 \times 10^{-4}$ at $5 \mathrm{~mA}$ in single bunch $1.1 \times 10^{-3}$

Peak Current [A] with $5 \mathrm{~mA} /$ bunch $\quad 12$ with $20 \mathrm{~mA} / \mathrm{bunch} \quad 31$

Horizontal emittance [nm rad]

$5 \mathrm{~mA} /$ bunch @ $700 \mathrm{MeV}<10$

$3 \mathrm{~mA} /$ bunch @ $500 \mathrm{MeV}<8$

Demonstrated FEL tunability [nm] 217-600

\section{MIRROR FEEDBACK SYSTEM}

To reduce power density and rate of degradation of a downstream mirror the Duke storage ring FEL has a very long optical cavity, which length is equal to the half of the ring circumference $(53.7 \mathrm{~m})$. To optimize the FEL gain the optical cavity has rather small Rayleigh range of $3.3 \mathrm{~m}$. Due to significant mismatch between Rayleigh range and the cavity length the OK-4/Duke FEL is especially susceptible to mirrors' vibrations and misalignments.

The 3 feet concrete slab with pillars going down to the bedrock provides stable and sturdy environment for the Duke storage ring and circulating electron beam. But the optical tables used for support of the mirror mounts are placed on the regular floor and, therefore, are prone to ambient vibrations. The tests showed that even changing of a static load on the optical table or to the floor nearby causes unacceptable changes of the mirror position. That is why an active feedback system for stabilizing mirrors, shown in the Fig. 1 was implemented.

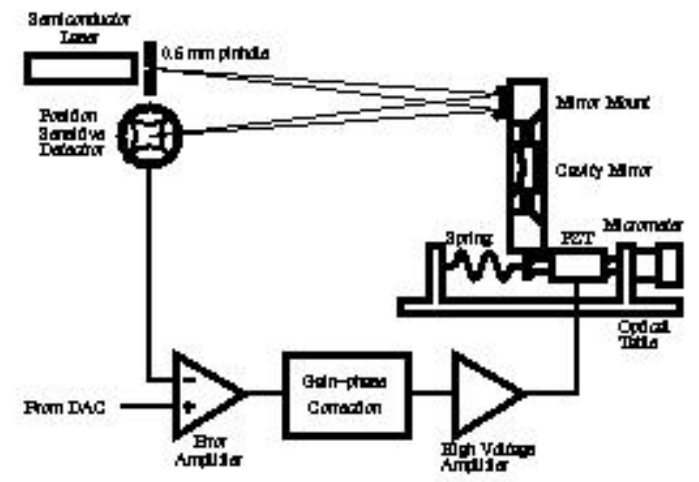

Figure 1: Active mirror feedback system

For this purpose two posts were installed inside the ring room, and reference semiconductor lasers and position sensitive detectors were mounted on the posts. The auxiliary concave mirror firmly attached to the optical cavity mirror mount reflects laser light. Tilt angle of the cavity mirror is measured by a position sensitive detector, which provides voltages proportional to the displacement of the light spot from the detector's center. The voltages from two digital-to-analog converters (DAC) set the desired tilt of the optical cavity mirror. The difference between the DAC voltage and detector voltage is used as an error signal in the feedback system. For the fast response the original stepper motors were replaced with the piezoelectric actuators, while coarse adjustment of the mirrors positions is performed with the help of manual micrometers.

From the optical mount inertia and piezoactuator stiffness the estimated cut-off frequency of feedback system can be up to $1 \mathrm{kHz}$. However, the mirror mount itself has a strong mechanical resonance at $100 \mathrm{~Hz}$. A gain-phase correction filter was implemented for 
providing stability of the closed loop system. After filter installation we were able to raise the cut-off frequency of the feedback system up to $50 \mathrm{~Hz}$ [3]. The vibrations with higher frequencies are suppressed with mechanical dampers incorporated to the optical table supports.

\section{GAIN MODULATOR}

The equilibrium between the energy spread growth, induced by the FEL power, quantum noise excitation and synchrotron damping set the limit on the average FEL power [4]. By modulating the optical cavity losses or the FEL gain, it is possible to compress the same level of average power into a number of giant pulses. This mode of operation is best suited for the high peak FEL power experiments.

The modulation of the optical cavity losses encounters a lot of technical difficulties: relatively low storage ring based FEL gain, high vacuum requirements. However, a modulation of the FEL gain by displacing the electron beam from the optical cavity axis can achieve the same results. Since the electron beam has negligible inertia, by using a set of fast steering magnets it is possible to switch on FEL instantaneously. More practical approach is to use a single corrector, providing adiabatic transition of the electron beam to the lasing orbit (adiabatic transition is required to avoid the excitation the electron beam oscillations). The Duke storage ring has tune frequencies of $700-900 \mathrm{kHz}$, and this requires a transition time of tens of microseconds.

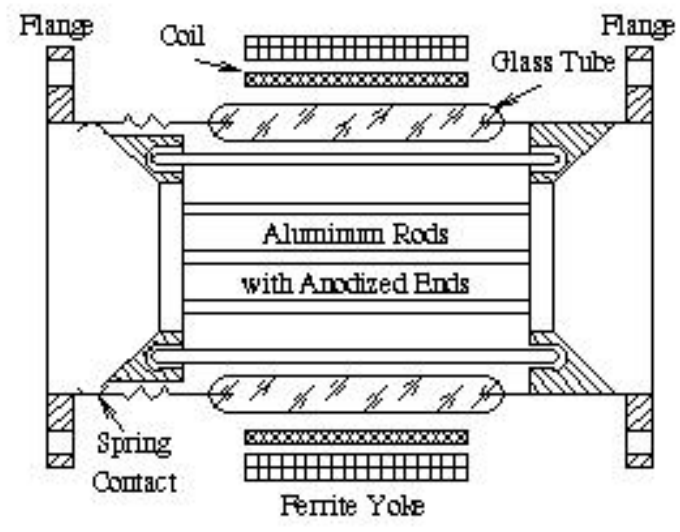

Figure 2: Gain modulator

The OK-4/Duke FEL gain modulator comprises a fast steering magnet with a ferrite yoke, control electronics, and a specially designed vacuum chamber (see Fig. 2). The vacuum chamber allows fast changes of the magnetic field and simultaneously provides smooth transition for the wake fields induced by the electron beam. Inside a glass tube, coated with a thin chromium layer, a cage of the aluminum rods is placed. The ends of the aluminum rods are anodized to provide capacitive coupling for the currents induced by the electron beam and to prevent eddy currents caused by the changing magnetic field.

In Fig. 3 two oscilloscope traces show the gain modulator operation. The first trace is the voltage from a probe coil placed inside gain modulator, this voltage is proportional to the first derivative of the magnetic field. The second one is integral of the coil voltage and it shows the changes of the magnetic field inside of gain modulator. The time constant of the gain modulator was chosen equal to 10 microseconds, what is much greater then betathron oscillation period and much less then FEL optical power time constant.

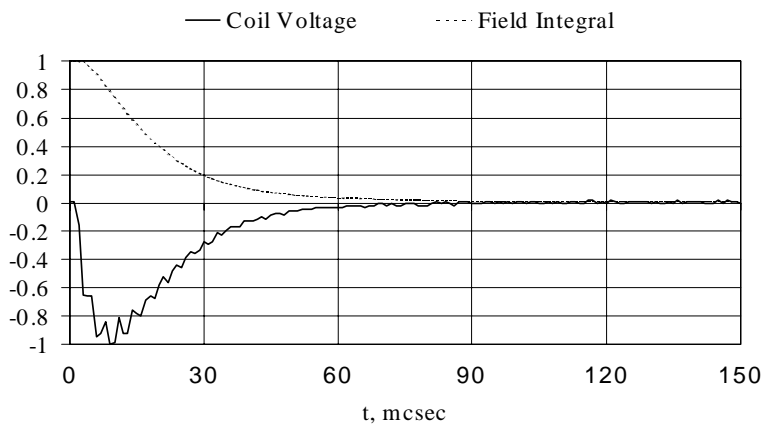

Figure 3: Signal from probe coil and field integral

Tests show that displacement of electron beam inside OK-4/Duke FEL is few millimeters in horizontal direction and less then 20 microns in vertical direction.

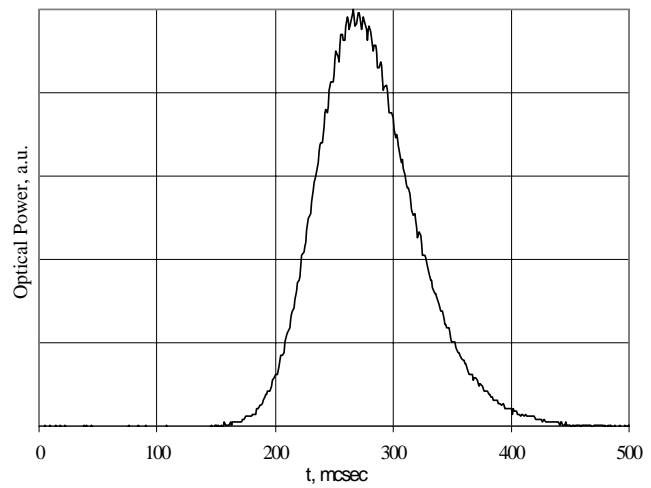

Figure 4: Giant pulse

The second trace is proportional to the optical power outcoupled from the OK-4/Duke FEL and measured with silicon photodiode.

\section{CONCLUSION}

Implementation of the optical cavity control system allowed to demonstrate stable lasing in the deep UV and visible range of the OK-4/Duke FEL. In future we want 
to replace existing mirror mounts for a mirror "switchyard", what will permit fast interchange of mirror sets for various wavelength and increase of mirror feedback bandwidth.

The gain modulator proved its essential role for medical user applications such as laser refractive surgery and no future upgrades are expected. It will be also used for the studying of FEL dynamical behavior.

Authors thank all the Duke FEL Laboratory staff for the efforts, which made successful operation of OK4/Duke FEL possible.

This work was supported by Office of Naval Research under the contract \#N00014-94-C0818.

\section{REFERENCES}

[1] V.N. Litvinenko et al., "OK-4/Duke Storage Ring FEL Lasing in the Deep UV." To be published in the Proceedings of the 18th International FEL Conference, Williamsburg, USA.

[2] Y. Wu et al., " The Performance of the Duke FEL Storage Ring." Nucl. Istr. and Meth., v. A375 (1996), pp. 74-77.

[3] I.V. Pinayev et al., "System for the Control and Stabilizing of OK4/Duke FEL Optical Cavity." AIP Proc. of 8th Beam Instrumentation Workshop, May 4-7, 1998, SLAC, Palo Alto, California, USA, pp. 545-551

[4] N.A. Vinokurov and A.N. Skrinsky, "Power Limit for an Optical Klystron Installed on Storage Ring", Preprint INP 77-59; A. Renieri, Nuovo Cimento 53B (1979), p. 160. 